\title{
Clinical value of color Doppler ultrasound combined with serum tumor markers for the diagnosis of medullary thyroid carcinoma
}

\author{
XUE YANG ${ }^{1}$, JINJUAN XU ${ }^{2}$, JILAN SUN $^{3}$, LIZHI YIN $^{4}$, RUI GUO ${ }^{5}$ and ZHIMEI YAN ${ }^{1}$ \\ ${ }^{1}$ Department of Ultrasound, The Affiliated Hospital of Qingdao University, Qingdao, Shandong 266000; \\ ${ }^{2}$ Physical Examination Department, People's Hospital of Rizhao, Rizhao, Shandong 276800; \\ ${ }^{3}$ Sterilization Supply Room, ${ }^{4}$ Health Management Center, ${ }^{5}$ Outpatient Department, \\ The People's Hospital of Zhangqiu Area, Jinan, Shandong 250200, P.R. China
}

Received June 2, 2020; Accepted April 8, 2021

DOI: 10.3892/ol.2021.12822

\begin{abstract}
The present study aimed to explore the clinical value of color Doppler ultrasound combined with serum tumor markers, including calcitonin $(\mathrm{CT})$ and carcinoembryonic antigen (CEA), for the diagnosis of medullary thyroid carcinoma (MTC). A total of 39 patients with MTC (MTC group), 50 patients with papillary thyroid carcinoma (PTC) (PTC group) and 30 patients with thyroid adenoma (benign control group) were enrolled in the present study. The patients were hospitalized at the Affiliated Hospital of Qingdao University from January 2012 to December 2018 and were diagnosed through surgical procedures and pathology laboratory results. The ultrasound results, as well as serum CT and CEA results, were collected and analyzed. A significant difference was observed between the MTC and PTC groups in regards to morphology, margin, aspect ratio, calcification, internal blood flow and lymph node metastasis (all $\mathrm{P}<0.01$ ). There was also a significant difference between the MTC and benign control group in regards to internal echo, calcification, internal blood flow and lymph node metastasis (all $\mathrm{P}<0.01$ ). In addition, the levels of serum CT and CEA in the MTC group were significantly higher than those in the PTC and the benign control groups (both $\mathrm{P}<0.01$ ). For patients with MTC, the levels of serum CT and CEA were significantly associated with maximum tumor diameter, lymph node metastasis and the patient state after treatment (all $\mathrm{P}<0.01)$. Furthermore, the sensitivities of ultrasound, serum CT and CEA for the diagnosis of MTC were $76.92,74.36$ and $68.23 \%$, respectively. The value for the combination of the three markers $(94.87 \%)$ was significantly higher compared with the sensitivity value of each separate marker (all $\mathrm{P}<0.05$ ). In conclusion, color Doppler
\end{abstract}

Correspondence to: Dr Zhimei Yan, Department of Ultrasound, The Affiliated Hospital of Qingdao University, 1677 Wutaishan Road, Huangdao, Qingdao, Shandong 266000, P.R. China

E-mail: ziwk97@163.com

Key words: medullary thyroid carcinoma, color Doppler ultrasound, serum calcitonin, carcinoembryonic antigen, joint examination ultrasound combined with detecting the levels of serum tumor markers (CT and CEA) significantly improved the diagnostic efficiency for MTC, which could be useful for the clinical diagnosis and treatment of MTC.

\section{Introduction}

Medullary thyroid carcinoma (MTC) is a malignant tumor derived from thyroid parafollicular cells (C cells) $(1,2)$. Hazard et al (3) first reported the disease in 1959. MTC is more common among women and middle-aged individuals. MTC is characterized by local infiltration and growth, and early lymphatic metastasis to the blood channel (4). Clinically, MTC can be classified into hereditary (20-30\% of cases) and sporadic (70-80\% of cases) types (5). MTC is a relatively rare type of thyroid cancer, accounting for 3-5\% of all thyroid tumors, with a mortality rate of $13.4 \%$ (6). The clinical incidence of MTC is relatively low; however, its malignancy is higher than that of differentiated thyroid carcinoma, between the severity of papillary carcinoma and undifferentiated carcinoma. MTC is an intermediate-grade malignant tumor with rapid progression, high recurrence rate, high metastasis rate and a poor prognosis. Up to $70 \%$ of patients with MTC have already suffered from cervical lymph node metastasis when the disease invades the thyroid nodule. Among these patients, about $10 \%$ have also already suffered from distant metastasis $(7,8)$. Therefore, early detection and timely treatment are crucial. Color Doppler ultrasound is the clinically preferred imaging examination tool for MTC. As a convenient and non-invasive diagnostic method, Color Doppler ultrasound plays an irreplaceable role in the early diagnosis and late follow-up of patients with MTC (9). However, ultrasound, as the most widely used method in the diagnosis of MTC, has several limitations. Sometimes it is not easy to differentiate papillary thyroid carcinoma (PTC) from thyroid adenoma, as the sensitivity is not favorable. Fine-needle aspiration (FNA) is used as the gold standard for the preoperative diagnosis of MTC; however, the sensitivity of FNA cytology alone for the diagnosis of MTC may be relatively low due to different sampling methods and angles (10). The rate of misdiagnosis and missed diagnosis is high (11). MTC originates from C cells that can secrete a variety of neuroendocrine polypeptides, 
such as calcitonin (CT) and carcinoembryonic antigen (CEA), therefore CT and CEA can be adopted as tumor markers. In fact, CT and CEA have been found to have crucial value in the clinical auxiliary diagnosis, assessment and monitoring of MTC $(12,13)$. In the present study, color Doppler ultrasound was used in combination with the detection of serum CT and CEA levels for the diagnosis of MTC, and the clinical value of the combined detection was determined.

\section{Patients and methods}

Clinical subjects. A total of 39 patients with MTC were enrolled into the MTC group. The patients were hospitalized at the Affiliated Hospital of Qingdao University (Qingdao, Shandong, China) from January 2012 to December 2018. They were diagnosed and verified through surgical procedures and pathology laboratory tests. The inclusion criteria were as follows: i) Patients diagnosed with sporadic MTC; ii) patients with complete color Doppler ultrasound and serum CT and CEA examination data; iii) patients without a history of malignant tumors in other organs other than the thyroid gland; iv) patients who had signed informed consent forms; v) patients who cooperated with researchers. The exclusion criteria were as follows: i) Patients with hereditary MTC, pheochromocytoma, or comorbid malignant tumors in other organs besides the thyroid gland; ii) patients without complete clinical data, and those who did not wish to be included in the study; iii) patients with other diseases, such as liver and kidney dysfunction, cardiovascular disease and diabetes; and iv) patients who were smokers or pregnant. In addition, 50 patients with PTC were enrolled into the PTC group, and 30 patients with thyroid adenoma were enrolled into the benign control group. Clinicopathological data of the study subjects in the three groups are presented in Table I. There were no significant differences in the clinicopathological variables between the three groups $(\mathrm{P}>0.05)$, and subjects in the three groups were comparable. The present study was approved by the Medical Ethics Committee of The Affiliated Hospital of Qingdao University (LIhao:201219) and all participants signed informed consent forms.

Examination methods. For 1.2.1 Color Doppler ultrasound examination, a PHILIPS iU22, color Doppler ultrasonic diagnostic apparatus, was used in the present study, with a linear array probe at a frequency of 7.0-15.0 MHz. During the examination, patients were asked to lie in the supine position, with their shoulders raised with pads, their heads tilted backwards and their necks extended. First, two-dimensional ultrasound was used to examine the thyroid glands. The size, morphology and capsule of the thyroid gland were observed. The location, shape, boundary, size, echo type and calcification of the lesion were analyzed. In addition, the lymph nodes around the thyroid gland were examined, particularly the bilateral cervical lymph nodes, to observe the number, size, shape and internal echo of the lymph nodes. Lymph node metastasis was analyzed and recorded. Subsequently, the blood flow signals in and around the lesion were examined with color Doppler flow imaging (CDFI).

Determination of serum CT and CEA levels. For specimen collection and pre-treatment, fasting elbow venous blood $(4 \mathrm{ml})$ was collected from each patient in the MTC group at 7:00 a.m. prior to surgery and 3 months after surgery. Blood samples were also collected from patients in the PTC group and the benign control group at 7:00 a.m. Samples were coagulated at room temperature $\left(25^{\circ} \mathrm{C}\right)$, followed by centrifugation at $2,264 \mathrm{x} \mathrm{g}$ for $20 \mathrm{~min}$ to separate the upper serum. Lipemia and hemolysis were excluded.

Serum CT and CEA levels were measured using the electrochemiluminescence method based on the double-antibody sandwich principle. A Cobas e601 full-automatic chemiluminescence immunoassay system from Roche Diagnostics was used. The reagents were provided by Roche Diagnostics (CEA, cat. no. 11731629322; CT, cat. no. 06445853190). All detection procedures were strictly performed in accordance with the kit instructions provided by the manufacturer, and the quality control met the requirements.

Categorization of findings. Pathological diagnosis was the gold standard. Consistencies between ultrasound examination and pathological diagnosis were defined as true positives, while inconsistencies between misdiagnosis, missed diagnosis and uncertainty were defined as false negatives. In the control group, consistencies between ultrasound examination and pathological diagnosis were defined as true negatives, while inconsistencies between them (misdiagnosis and uncertainty) were defined as false positives. Serum CT and CEA levels exceeding the cut-off levels were positive, and serum CT and CEA levels lower than or equal to the cut-off levels were negative. The joint examinations had a positive outcome if one or more detection results were positive. If all detection results were negative, the joint examinations could be regarded as negative.

Statistical analysis. For statistical analysis, SPSS 19.0 (IBM Corp.) was used. Measurement data exhibited a skewed distribution, and were expressed as the median and quartile range [M (25-75\%)]. The non-parametric Kruskal-Wallis H test was used for comparisons among multiple groups, while the Mann-Whitney U test or Bonferroni test was used for pairwise comparisons between two groups. Enumeration data were analyzed using the $\chi^{2}$ test. With the thyroid adenoma group as a control, receiver operating characteristic (ROC) curves of the CT and CEA of the participants were drawn, and the areas under the curves (AUC) were calculated. The optimal cut-off values for CT and CEA for the diagnosis of MTC were determined according to the maximum Youden indexes. Taking pathological diagnosis as the gold standard, the examination results were true positive (a), false positive (b), false negative (c) and true negative (d). The formula used for calculation was as follows: Sensitivity $=a /(a+c)$; specificity $=d /(d+b)$; accuracy $=(a$ $+\mathrm{d}) /(\mathrm{a}+\mathrm{b}+\mathrm{c}+\mathrm{d}) ;$ positive predictive value $=\mathrm{a} /(\mathrm{a}+\mathrm{b})$; negative predictive value $=\mathrm{d} /(\mathrm{d}+\mathrm{c})$; positive likelihood $\mathrm{ratio}=(\mathrm{a} / \mathrm{a}+$ $\mathrm{c}) /(\mathrm{b} / \mathrm{b}+\mathrm{d})$; and negative likelihood ratio $=(\mathrm{c} / \mathrm{a}+\mathrm{c}) /(\mathrm{d} / \mathrm{b}+\mathrm{d})$. $\mathrm{P}<0.05$ indicated a statistically significant difference.

\section{Results}

Comparison of color Doppler ultrasonographic features among MTC, PTC and thyroid adenoma. CDFI of MTC samples revealed that MTC samples had a clear boundary, regular shape (oval or round), an aspect ratio of $\leq 1$, hypoecho or extreme 
Table I. Comparison of the general information of subjects in the three groups.

\begin{tabular}{|c|c|c|c|c|c|}
\hline Features & MTC group $(n=39)$ & PTC group $(n=50)$ & Benign control group & $\chi^{2}$ & P-value \\
\hline \multicolumn{6}{|l|}{ Sex } \\
\hline Male & 21 & 24 & 14 & 0.436 & 0.804 \\
\hline Female & 18 & 26 & 16 & & \\
\hline \multicolumn{6}{|l|}{ Age (years) } \\
\hline$\leq 40$ & 15 & 20 & 9 & 0.860 & 0.651 \\
\hline$>40$ & 24 & 30 & 21 & & \\
\hline \multicolumn{6}{|l|}{ Marriage status } \\
\hline Unmarried & 7 & 11 & 4 & 0.946 & 0.623 \\
\hline Married & 32 & 39 & 26 & & \\
\hline \multicolumn{6}{|l|}{ Education } \\
\hline High school degree and below & 23 & 32 & 17 & 0.479 & 0.787 \\
\hline College degree and above & 16 & 18 & 13 & & \\
\hline \multicolumn{6}{|l|}{ Income (Yuan) } \\
\hline$\leq 3000$ & 24 & 32 & 16 & 0.919 & 0.632 \\
\hline$>3000$ & 15 & 18 & 14 & & \\
\hline \multicolumn{6}{|l|}{ Place of residence } \\
\hline Coastal region & 12 & 13 & 8 & 0.271 & 0.873 \\
\hline Inland & 27 & 37 & 22 & & \\
\hline \multicolumn{6}{|l|}{ Occupation } \\
\hline Farmer & 11 & 13 & 8 & 0.288 & 0.991 \\
\hline Worker & 19 & 27 & 16 & & \\
\hline Cadres & 9 & 10 & 6 & & \\
\hline
\end{tabular}

MTC, medullary thyroid carcinoma; PTC, papillary thyroid carcinoma. Data are expressed as number of patients.

hypoecho, mostly coarse calcification, a rich and convoluted internal blood flow, and discontinuous peripheral blood flow signals (Fig. 1). CDFI of PTC samples demonstrated that PTC samples had an unclear boundary, irregular shape, an aspect ratio of $>1$, hypoecho or extreme hypoecho, mostly microcalcification, and rich or unrich internal blood flow signals (Fig. 2). CDFI of thyroid adenoma samples revealed that the thyroid adenoma samples had a clear boundary, regular shape (oval or round), an aspect ratio of $\leq 1$, hypoecho or extreme hypoecho, no calcification in the interior, neither rich nor unrich internal blood flow signals, and a regular course of peripheral blood flow signals (Fig. 3). The comparison of color Doppler ultrasonographic features between MTC and PTC samples is shown in Table II, and the comparison of the ultrasonic features between MTC and thyroid adenoma samples is presented in Table III.

Comparison of serum CT and CEA levels between the MTC, PTC and thyroid adenoma groups. The levels of serum CT and CEA in patients with MTC were significantly higher compared with those of patients with PTC and thyroid adenoma $(\mathrm{P}<0.01)$. However, there was no significant difference in the levels of serum CT and CEA between patients with PTC and those with thyroid adenoma $(\mathrm{P}>0.05$; Table IV).

Association between serum CT and CEA levels, and various clinicopathological features of patients with MTC. The serum levels of CT and CEA in patients with MTC were not significantly associated with sex and age (both $\mathrm{P}>0.05$ ); however, they were significantly associated with the maximum tumor diameter, lymph node metastasis and the state of the patients after treatment (all $\mathrm{P}<0.01$; Table $\mathrm{V}$ ).

Comparison of the diagnostic value of color Doppler ultrasound, and serum CT and CEA levels alone, and color Doppler ultrasound combined with serum CT and CEA levels for the diagnosis of MTC. With postoperative pathological diagnosis as the gold standard, among the 39 patients with MTC, consistencies between ultrasound examination and pathological diagnosis were found in 30 patients (true positive), while inconsistencies between the two diagnostic methods were detected in 9 patients (false negative). Among the 30 patients with thyroid adenoma, consistencies between ultrasound examination and pathological diagnosis were found in 28 patients (true negative), while inconsistencies was detected in 2 patients (false positive)

The areas under the ROC curves for CT and CEA levels in the diagnosis of MTC were 0.838 and 0.789 , respectively, (Figs. 4 and 5). The CT and CEA values corresponding to the maximum Youden indices of CT and CEA (0.675 and 0.641) were selected as the optimal critical values for the diagnosis of MTC. The critical values of CT and CEA were $6.70 \mathrm{pg} / \mathrm{ml}$ and $4.60 \mathrm{ng} / \mathrm{ml}$, respectively. The diagnostic values of color Doppler ultrasound, serum CT or serum CEA alone, and color Doppler ultrasound combined with serum CT and CEA for the 


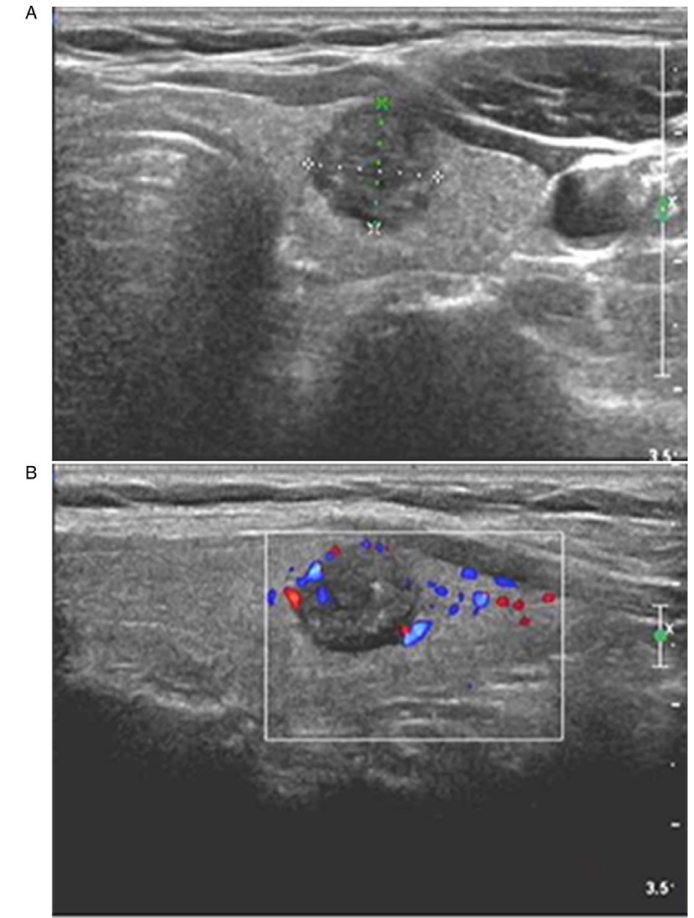

Figure 1. Diagnosis of MTC in a 42-year-old women through postoperative pathology. (A) Two-dimensional ultrasound showing a solitary nodule in the upper pole of the right lobe of thyroid gland, with an oval shape, an aspect ratio of $<1$, a clear boundary, hypoecho, and internal coarse calcification. (B) CDFI showing blood flow signals inside and around the nodule; the ultrasound results suggested that the nodule met the TI-RADS category 5 , and that it may be MTC. MTC, medullary thyroid carcinoma; CDFI, color Doppler flow imaging.

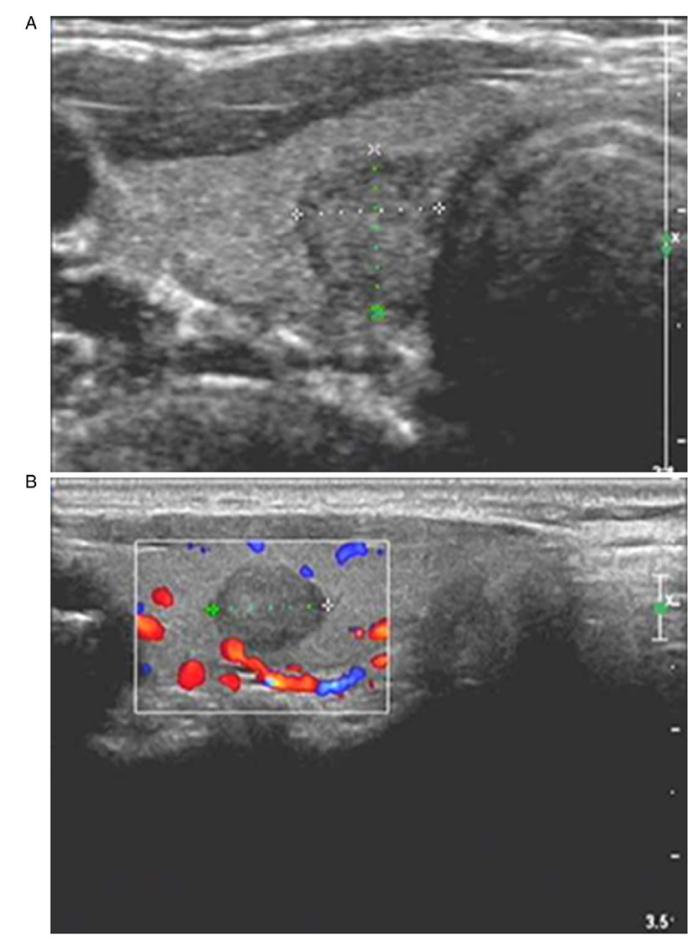

Figure 2. Diagnosis of PTC in a 39-year-old female through postoperative pathology. (A) Two-dimensional ultrasound showing a solitary nodule in the upper pole of the right lobe of thyroid gland, with an irregular shape, an aspect ratio of $>1$, an unclear boundary, hypoecho and internal microcalcification. (B) CDFI showing blood flow signals inside and around the nodule; the ultrasound results suggested that the nodule met the TI-RADS category 5 , and that it may be PTC. PTC, papillary thyroid carcinoma; CDFI, color Doppler flow imaging.

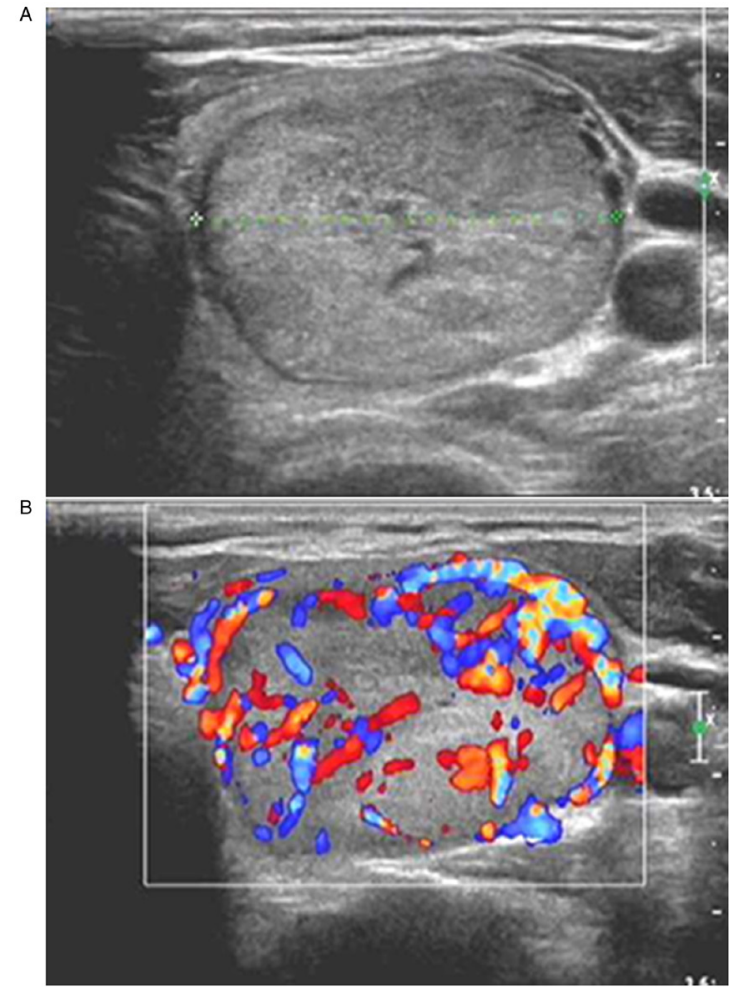

Figure 3. Diagnosis of thyroid adenoma in a 53-year-old male through postoperative pathology. (A) Two-dimensional ultrasound showing a solitary nodule in the middle lower pole of the left lobe of thyroid gland, with an oval shape, an aspect ratio of $<1$, clear boundary, isoecho and no internal calcification. (B) CDFI showing thick blood flow signals inside and around the nodule, and a regular course of peripheral blood flow signals; the ultrasound results suggested that the nodule met the TI-RADS category 3 , and that it may be thyroid adenoma. CDFI, color Doppler flow imaging.

diagnosis of MTC were analyzed (Table VI). Color Doppler ultrasound combined with serum CT and CEA levels exhibited a significantly higher sensitivity compared with color Doppler ultrasound, serum CT or serum CEA alone (all $\mathrm{P}<0.05$ ). The positive likelihood ratios of ultrasound, serum CT, serum CEA and the combined detection of MTC were 22.62, 22.54, 10.29 and 14.16 , respectively, and the negative likelihood ratios were $0.24,0.36,0.33$ and 0.06 , respectively.

\section{Discussion}

Medullary thyroid carcinoma (MTC) is a highly malignant tumor and prone to local spread or distant metastasis. It is crucial to find a correct early diagnosis prior to surgical intervention in order to make an appropriate surgical scheme to avoid a second surgery and improve the survival rate (14). It has been shown that the proto-oncogene RET mutation is the main pathogenic factor in MTC and can be used to detect MTC (15). However, this method has several limitations, such as a high cost, poor diagnostic yield in patients with sporadic MTC. And it is not able to differentiate the various subtypes of MTC pathogenic genes. Thus, the detection of the gene cannot be readily applied to clinical practice on a large scale. In addition, pre-operative fine-needle aspiration (FNA) has limitations due to its poor diagnostic positive rate and inability to evaluate lymph node metastasis. Therefore, researchers have been searching for economical, efficient 
Table II. Comparison of color Doppler ultrasonographic features between MTC and PTC.

\begin{tabular}{|c|c|c|c|c|}
\hline Ultrasonic imaging & $\operatorname{MTC}(n=39)$ & PTC $(n=50)$ & $\chi^{2}$ & P-value \\
\hline \multicolumn{5}{|l|}{ Position } \\
\hline Middle upper pole & 23 & 28 & 0.030 & $>0.05$ \\
\hline Lower pole & 16 & 21 & & \\
\hline \multicolumn{5}{|l|}{ Echo } \\
\hline Hypoecho or extreme hypoecho & 37 & 44 & 1.265 & $>0.05$ \\
\hline Isoecho or hyperecho & 2 & 6 & & \\
\hline \multicolumn{5}{|l|}{ Boundary } \\
\hline Clear & 24 & 13 & 11.392 & $<0.01$ \\
\hline Unclear & 15 & 37 & & \\
\hline \multicolumn{5}{|l|}{ Shape } \\
\hline Round or oval & 25 & 12 & 14.507 & $<0.01$ \\
\hline Irregular & 14 & 38 & & \\
\hline \multicolumn{5}{|l|}{ Aspect ratio } \\
\hline$>1$ & 6 & 32 & 21.165 & $<0.01$ \\
\hline$\leq 1$ & 33 & 18 & & \\
\hline \multicolumn{5}{|l|}{ Calcification } \\
\hline No & 2 & 10 & 24.128 & $<0.01$ \\
\hline Microcalcification & 11 & 32 & & \\
\hline Course calcification & 26 & 8 & & \\
\hline \multicolumn{5}{|l|}{ Internal blood flow } \\
\hline No & 3 & 5 & 13.390 & $<0.01$ \\
\hline Not rich & 11 & 32 & & \\
\hline Rich & 25 & 13 & & \\
\hline \multicolumn{5}{|l|}{ Lymph node metastasis } \\
\hline No & 21 & 43 & 11.214 & $<0.01$ \\
\hline Yes & 18 & 7 & & \\
\hline
\end{tabular}

MTC, medullary thyroid carcinoma; PTC, papillary thyroid carcinoma. Data are expressed as number of patients.

and specific indicators and methods for the pre-operative evaluation of MTC.

At present, neck ultrasonography is the preferred diagnostic method for MTC. Under ultrasound, MTC can be preliminarily diagnosed by comprehensively analyzing features, including morphology, boundary, aspect ratio, internal components, blood flow signals and the cervical lymph nodes of thyroid nodules. Ultrasonic signs of MTC have a few common features with those of papillary thyroid carcinoma (PTC) and thyroid adenoma. MTC is generally round or oval in shape, similar to thyroid tumors, with an aspect ratio of $\leq 1$. Lee et al (16) discovered that about $67.40 \%$ of MTC nodules were round or oval, and only $13 \%$ of nodules had an aspect ratio of $>$ in MTC. Kim et al (17) came to a similar conclusion based on the following clinical observation: The boundary of MTC nodules was mostly clear, similar to benign lesions (18), while the boundary of PTC nodules was irregular, unclear or burr-like. Calcification is considered an important sign of a thyroid malignant tumor (19). Coarse calcification is common in MTC, which is related to amyloid deposition in the intercellular substances of $\mathrm{C}$ cells. Microcalcification is common in PTC, whereas thyroid adenoma usually exhibits no calcification. Intranodular hypoecho and extreme hypoecho are common manifestations of MTC and PTC ultrasound images (20). In the present study, it was demonstrated that among patients with MTC, $64.10 \%$ of tumors had a round or oval nodule, $61.54 \%$ had a clear boundary, and $84.62 \%$ had an aspect ratio $\leq 1$, which is consistent with the findings of previous studies. In addition, $66.67 \%$ of patients in the MTC group had tumors with course calcification, which was relatively higher than the results reported by Choi et al (19), which may be related to the method used for the patient selection process. There were 32 patients $(64.00 \%)$ with microcalcification in the PTC group and 25 patients $(83.33 \%)$ without calcification in the benign control group. The blood flow signal characteristics of nodules are of great value in judging their benign and malignant properties. Compared with PTC, the blood flow signal shown in MTC ultrasound is richer (21). In addition, under color Doppler ultrasound, the characteristics of blood flow signals between MTC and thyroid adenoma are often similar; that is, both are surrounded by the peripheral blood flow signals of nodules. However, differences between them can be found through careful observation. The peripheral 
Table III. Comparison of the ultrasonic features between MTC and thyroid adenoma.

\begin{tabular}{|c|c|c|c|c|}
\hline Ultrasonic imaging & MTC $(n=39)$ & Thyroid adenoma $(n=30)$ & $\chi^{2}$ & P-value \\
\hline \multicolumn{5}{|l|}{ Position } \\
\hline Middle upper pole & 23 & 18 & 0.007 & $>0.05$ \\
\hline Lower pole & 16 & 12 & & \\
\hline \multicolumn{5}{|l|}{ Echo } \\
\hline Hypoecho or extreme hypoecho & 37 & 19 & 11.030 & $<0.01$ \\
\hline Isoecho or hyperecho & 2 & 11 & & \\
\hline \multicolumn{5}{|l|}{ Boundary } \\
\hline Clear & 24 & 20 & 0.193 & $>0.05$ \\
\hline Unclear & 15 & 10 & & \\
\hline \multicolumn{5}{|l|}{ Shape } \\
\hline Round or oval & 25 & 23 & 0.361 & $>0.05$ \\
\hline Irregular & 14 & 17 & & \\
\hline \multicolumn{5}{|l|}{ Aspect ratio } \\
\hline$>1$ & 6 & 3 & 0.433 & $>0.05$ \\
\hline$\leq 1$ & 33 & 27 & & \\
\hline \multicolumn{5}{|l|}{ Calcification } \\
\hline No & 2 & 25 & 45.609 & $<0.01$ \\
\hline Microcalcification & 11 & 4 & & \\
\hline Course calcification & 26 & 1 & & \\
\hline \multicolumn{5}{|l|}{ Internal blood flow } \\
\hline No & 3 & 8 & 15.99 & $<0.01$ \\
\hline Not rich & 11 & 17 & & \\
\hline Rich & 25 & 5 & & \\
\hline \multicolumn{5}{|l|}{ Lymph node metastasis } \\
\hline No & 21 & 30 & 18.733 & $<0.01$ \\
\hline Yes & 18 & 0 & & \\
\hline
\end{tabular}

MTC, medullary thyroid carcinoma. Data are expressed as number of patients.

Table IV. Comparison of the levels of serum CT and CEA among the three groups.

\begin{tabular}{|c|c|c|c|c|c|c|c|}
\hline Groups & $\mathrm{N}$ & $\mathrm{CT}(\mathrm{pg} / \mathrm{ml})$ & $\mathrm{U}$ & P-value & CEA (ng/ml) & $\mathrm{U}$ & P-value \\
\hline MTC group & 39 & $345.35(69.34,1123.44)$ & & & $42.75(10.67,102.45)$ & & \\
\hline PTC group & 50 & $3.12(1.79,4.12)^{\mathrm{a}}$ & 256.500 & $<0.01$ & $2.79(1.38,3.76)^{\mathrm{a}}$ & 288.000 & $<0.01$ \\
\hline Benign control group & 30 & $2.98(1.15,3.98)^{\mathrm{b}}$ & 178.000 & $<0.01$ & $2.64(1.07,3.57)^{\mathrm{b}}$ & 139.000 & $<0.01$ \\
\hline
\end{tabular}

${ }^{\mathrm{a}} \mathrm{P}<0.01$ vs. the MTC group; ${ }^{\mathrm{b}} \mathrm{P}<0.01$ vs. the MTC group. CT, calcitonin; CEA, carcinoembryonic antigen; MTC, medullary thyroid carcinoma; PTC, papillary thyroid carcinoma. Data are expressed as [mean (25-75\%)].

blood flow around MTC is discontinuous, while adenoma can surround the tumor by $1 / 2$ or more, and the course of blood vessels is regular (22). The correct diagnosis of MTC under ultrasound requires a comprehensive judgment based on nodule morphology, edge, aspect ratio, calcification and blood flow. In the present study, the sensitivity of ultrasonic diagnosis of MTC was unsatisfactory (76.92\%); consequently, other examinations had to be used along with ultrasonic diagnosis to improve the diagnostic value.
The application value of calcitonin (CT) has been evaluated by a series of prospective non-randomized studies. Routine serum CT screening can be adopted to detect the early proliferation of C cells and MTC, thus improving the detection rate of MTC $(23,24)$. CT scans have a very high accuracy to rule out the possibility of MTC if the serum CT levels are lower than the reference upper limit (25). The carcinoembryonic antigen (CEA) level also has an important reference value for the diagnosis of thyroid cancer (26), and it is more valuable 
Table V. Relationship between serum CT and CEA levels and different clinicopathological features of the patients with MTC.

\begin{tabular}{|c|c|c|c|c|c|c|c|}
\hline Groups & $\mathrm{N}$ & CT (pg/ml) & $\mathrm{U}$ & P-value & CEA (ng/ml) & $\mathrm{U}$ & P-value \\
\hline \multicolumn{8}{|l|}{ Sex } \\
\hline Male & 21 & $362.19(58.86,1210.45)$ & \multirow[t]{2}{*}{425.300} & \multirow[t]{2}{*}{$>0.05$} & $46.67(8.12,114.61)$ & \multirow[t]{2}{*}{466.900} & \multirow[t]{2}{*}{$>0.05$} \\
\hline Female & 28 & $329.24(75.67,1015.36)$ & & & $39.82(11.23,98.67)$ & & \\
\hline \multicolumn{8}{|l|}{ Age (years) } \\
\hline$\leq 50$ & 23 & $327.16(81.43,986.75)$ & \multirow[t]{2}{*}{619.200} & \multirow[t]{2}{*}{$>0.05$} & $37.54(12.36,96.34)$ & \multirow[t]{2}{*}{568.300} & \multirow[t]{2}{*}{$>0.05$} \\
\hline$>50$ & 16 & $359.78(61.43,1225.34)$ & & & $47.61(9.13,116.78)$ & & \\
\hline \multicolumn{8}{|c|}{ Tumor diameter (cm) } \\
\hline$\leq 1$ & 12 & $102.33(31.42,186.31)$ & \multirow[t]{2}{*}{89.000} & \multirow[t]{2}{*}{$<0.01$} & $26.54(6.79,35.27)$ & \multirow[t]{2}{*}{93.000} & \multirow[t]{2}{*}{$<0.01$} \\
\hline$>1$ & 27 & $489.43(125.42,1361.27)$ & & & $68.79(37.68,120.67)$ & & \\
\hline \multicolumn{8}{|c|}{ Lymph node metastasis } \\
\hline Yes & 18 & $782.34(213.56,1364.21)$ & \multirow[t]{2}{*}{102.000} & \multirow[t]{2}{*}{$<0.01$} & $86.79(43.22,33.24)$ & \multirow[t]{2}{*}{113.000} & \multirow[t]{2}{*}{$<0.01$} \\
\hline No & 21 & $95.13(21.56,159.45)$ & & & $18.67(7.64,32.77)$ & & \\
\hline \multicolumn{8}{|c|}{ Before and after treatment } \\
\hline Before treatment & 39 & $345.35(69.34,1123.44)$ & \multirow[t]{2}{*}{236.000} & \multirow[t]{2}{*}{$<0.01$} & $42.75(10.67,102.45)$ & \multirow[t]{2}{*}{201.000} & \multirow[t]{2}{*}{$<0.01$} \\
\hline After treatment & 39 & $5.26(2.63,7.34)$ & & & $4.32(2.17,5.25)$ & & \\
\hline
\end{tabular}

CT, calcitonin; CEA, carcinoembryonic antigen; MTC, medullary thyroid carcinoma. Data are expresses as [mean (25-75\%)].

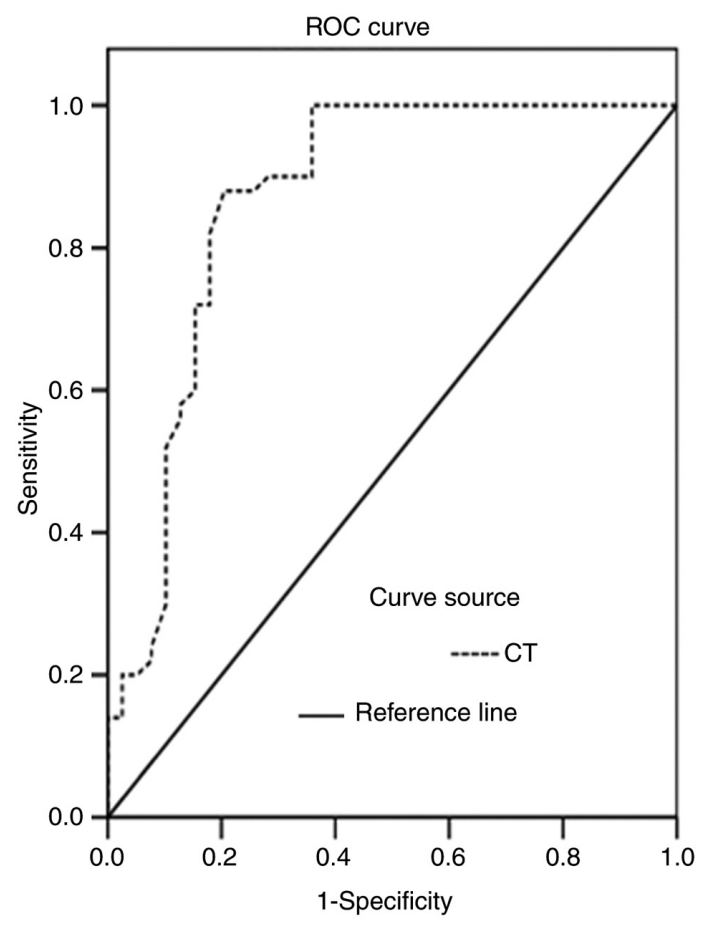

Figure 4. ROC curve of serum CT levels for the diagnosis of MTC. MTC, medullary thyroid carcinoma; ROC, receiver operating characteristic; $\mathrm{CT}$, calcitonin.

when serum CT and CEA levels increase simultaneously. As characteristic secretion products of MTC, serum CT and CEA levels are significantly increased in the majority of cases with MTC (27), which was also verified in the present study. In the present study, it was also demonstrated that the levels of serum CT and CEA in the MTC group were significantly higher compared with those in the PTC and benign control groups.

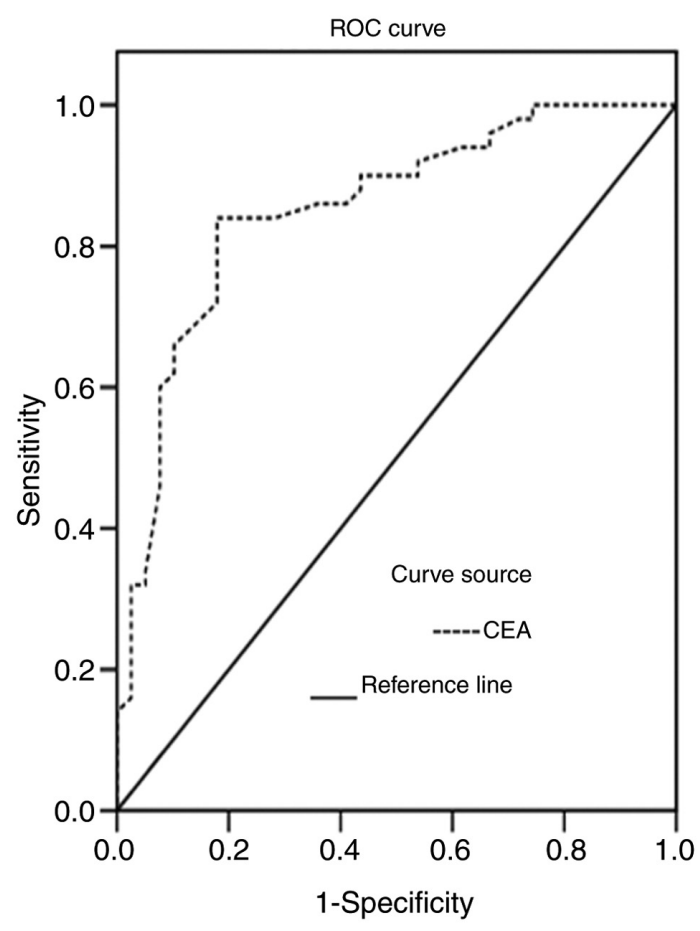

Figure 5. ROC curve of serum CEA levels for the diagnosis of MTC. MTC, medullary thyroid carcinoma; CEA, carcinoembryonic antigen; ROC, receiver operating characteristic.

Among the 39 patients with MTC, 29 had increased levels of CT, and 27 had elevated levels of CEA. Of course, this does not mean that all patients with elevated serum CT necessarily suffer from MTC. Equally, normal levels of serum CT and CEA cannot rule out the possibility of MTC. The accurate diagnosis of MTC can be achieved by combining the CT scan and CEA level results with other imaging techniques, such as ultrasonic 
Table VI. Comparison of the diagnostic value of color Doppler ultrasound, serum CT, serum CEA, and color Doppler ultrasound combined with serum CT and CEA in diagnosing MTC [\% (n)].

\begin{tabular}{lccccc}
\hline Detection indices & Sensitivity & Specificity & Accuracy & $\begin{array}{c}\text { Predictive } \\
\text { positive value }\end{array}$ & $\begin{array}{c}\text { Predictive } \\
\text { negative value }\end{array}$ \\
\hline Ultrasound & $76.92(30 / 39)$ & $96.67(29 / 30)$ & $84.06(58 / 69)$ & $93.75(30 / 32)$ & $75.68(28 / 37)$ \\
CT & $74.36(29 / 39)$ & $96.67(29 / 30)$ & $84.06(58 / 69)$ & $96.67(29 / 30)$ & $72.50(29 / 40)$ \\
CEA & $68.23(27 / 39)$ & $93.33(28 / 30)$ & $78.26(54 / 69)$ & $90.00(27 / 30)$ & $69.23(27 / 39)$ \\
Ultrasound combined with & $94.87(37 / 39)^{\mathrm{a}}$ & $93.33(28 / 30)$ & $91.30(63 / 69)$ & $90.24(37 / 41)$ & $92.86(26 / 28)$ \\
CT and CEA & & & & & \\
$\chi^{2}$ & 8.724 & 0.702 & 4.490 & 1.391 & 5.482 \\
P-value & 0.033 & 0.873 & 0.213 & 0.708 & 0.140 \\
\hline
\end{tabular}

${ }^{\mathrm{a}} \mathrm{P}<0.05$ vs. the separate ultrasound, serum CT, and serum CEA. CT, calcitonin; CEA, carcinoembryonic antigen; MTC, medullary thyroid carcinoma.

characteristics. Serum CT and CEA levels can be helpful tools that may be used to evaluate the severity of the disease, and high levels of CT and CEA are indicative of a large tumor with lymph node metastasis (28). In the present study, CT and CEA levels in patients with tumor diameters of $>1 \mathrm{~cm}$ and those with lymph node metastasis were significantly higher compared with those in patients with tumor diameters $\leq 1 \mathrm{~cm}$ and those without lymph node metastasis. Therefore, serum CT and CEA levels were also prognostic predictors of MTC. Serum CT and CEA levels are also helpful for monitoring the recurrence and metastasis of MTC following surgery. There is at least one study demonstrating that the aforementioned indices can be used to reveal the probability of recurrence or metastasis prior to imaging tests (29). The dynamic observation of serum CT and CEA levels following surgery can play a crucial role in judging the effectiveness of the surgical intervention and risk of tumor recurrence (30). According to the ROC curves of CT and CEA serum levels, when CT and CEA values corresponding to the maximum Youden indices of CT (0.675) and CEA (0.641) were selected as the optimal cut-off values $(6.70 \mathrm{pg} / \mathrm{ml}$ and $4.60 \mathrm{ng} / \mathrm{ml}$, respectively) for the diagnosis of MTC, the sensitivity of CT and CEA for the diagnosis of MTC was 74.36 and $68.23 \%$, respectively. However, the sensitivity of spate examination was low. The occurrence of false positives and false negatives cannot be ruled out by in vitro diagnostic tests, and other methods need to be combined to improve the diagnostic accuracy.

The results of the present study demonstrated that the sensitivity of ultrasound combined with measuring serum CT and CEA levels for the diagnosis of MTC was 94.87\%, which was significantly higher than that of single tests $(\mathrm{P}<0.05)$. The positive and negative likelihood rations of combined detection were 14.16 and 0.06 , respectively. When the positive likelihood ratio was $>10$ or the negative likelihood ratio was $<0.1$, the possibility of diagnosing or excluding a certain disease was significantly increased.

It should be acknowledged that there are a few limitations to the present study. For example, the present study did not carry out a systematic follow-up on the long-term therapeutic effect. In the future, the authors aim to focus on the association between the changes in serum CT and CEA levels and the survival rate of patients.
In conclusion, ultrasound combined with measuring serum CT and CEA levels may be a helpful technique for the early detection of MTC and was shown to be effective in reducing the missed diagnosis rate. Nonetheless, the specificity of the combined examination was reduced. Therefore, it remains necessary to rely on pathological morphology, immunohistochemistry and specific staining technique to confirm the diagnosis. The detection of RET gene mutation can provide an early genetic diagnosis of MTC, which is the future developmental direction.

\section{Acknowledgements}

Not applicable.

\section{Funding}

No funding was received.

\section{Availability of data and materials}

The datasets used and/or analyzed during the current study are available from the corresponding author on reasonable request.

\section{Authors' contributions}

XY designed the study and drafted the manuscript. JX and JS were responsible for the collection and analysis of the experimental data. LY, RG and ZY performed Color Doppler ultrasound examination and revised the manuscript critically for important intellectual content. All authors read and approved the final manuscript.

\section{Ethics approval and consent to participate}

The study was approved by the Ethics Committee of The Affiliated Hospital of Qingdao University (LIhao:201219), China. Patients who participated in this research, signed the informed consent and had complete clinical data. Signed written informed consents were obtained from the patients and/or guardians. 


\section{Patient consent for publication}

Not applicable.

\section{Competing interests}

The authors declare that they have no competing interests.

\section{References}

1. Mohammadi M and Hedayati M: A brief review on the molecular basis of medullary thyroid carcinoma. Cell J 18: 485-492, 2017.

2. Bae YJ, Schaab M and Kratzsch J: Calcitonin as biomarker for the medullary thyroid carcinoma. Recent Results Cancer Res 204: 117-137, 2015

3. Hazard JB, Hawk WA and Crile G Jr: Medullary (solid) carcinoma of the thyroid; a clinicopathologic entity. J Clin Endocrinol Metab 19: 152-161, 1959.

4. Elisei R, Alevizaki M, Conte-Devolx B, Frank-Raue K, Leite V and Williams GR: 2012 European thyroid association guidelines for genetic testing and its clinical consequences in medullary thyroid cancer. Eur Thyroid J 1: 216-231, 2013

5. Roman S, Lin R and Sosa JA: Prognosis of medullary thyroid carcinoma: Demographic, clinical, and pathologic predictors ofsurvival in 1252 cases. Cancer 107: 2134-2142, 2006.

6. Ferlay J, Colombet M, Soerjomataram I, Dyba T, Randi G, Bettio M, Gavin A, Visser O and Bray F: Cancer incidence and mortality patterns in Europe: Estimates for 40 countries and 25 major cancers in 2018. Eur J Cancer 103: 356-387, 2018.

7. Kebebew E, Ituarte PH, Siperstein AE, Duh QY and Clark OH: Medullary thyroid carcinoma: Clinical characteristics, treatment, prognostic factors, and a comparison of staging systems. Cancer 88: 1139-1148, 2000.

8. Grande E, Santamaría Sandi J, Capdevila J, Navarro González E, Zafón Llopis C, Ramón Y Cajal Asensio T, Gómez Sáez JM Jiménez-Fonseca P, Riesco-Eizaguirre G and Galofré JC: Consensus on management of advanced medullary thyroid carcinoma on behalf of the working group of thyroid cancer of the Spanish society of endocrinology (SEEN) and the Spanish task force group for orphan and infrequent tumors (GETHI) Clin Transl Oncol 18: 769-775, 2016.

9. Yang GC, Fried K and Levine PH: Detection of medullary thyroid microcarcinoma using ultrasound-guided fine needle aspiration cytology. Cytopathology 24: 92-98, 2013.

10. Essig GF Jr, Porter K, Schneider D, Debora A, Lindsey SC Busonero G, Fineberg D, Fruci B, Boelaert K, Smit JW, et al: Fine needle aspiration and medullary thyroid carcinoma: The risk of inadequate preoperative evaluation and initial surgery when relying upon FNAB cytology alone. Endocr Pract 19: 920-927, 2013

11. Trimboli P, Treglia G, Guidobaldi L, Romanelli F, Nigri G, Valabrega S, Sadeghi R, Crescenzi A, Faquin WC, Bongiovanni M and Giovanella L: Detection rate of FNA cytology in medullary thyroid carcinoma: A meta-analysis. Clin Endocrinol (Oxf) 82: 280-285, 2015

12. Jung KY, Kim SM, Yoo WS, Kim BW, Lee YS, Kim KW, Lee KE, Jeong JJ, Nam KH, Lee SH, et al: Postoperative biochemical remission of serum calcitonin is the best predictive factor for recurrence-free survival of medullary thyroid cancer: A large-scale retrospective analysis over 30 years. Clin Endocrinol (Oxf) 84: 587-597, 2016.

13. Raue F and Frank-Raue K: Long-term follow-up in medullary thyroid carcinoma. Recent Results Cancer Res 204: 207-225, 2015

14. Wells SA Jr, Asa SL, Dralle H, Elisei R, Evans DB, Gagel RF, Lee N, Machens A, Moley JF, Pacini F, et al: Revised American Thyroid Association guidelines for the management of medullary thyroid carcinoma. Thyroid 25: 567-610, 2015.

15. Romei C, Ciampi R and Elisei R: A comprehensive overview of the role of the RET proto-oncogene in thyroid carcinoma. Nat Rev Endocrinol 12: 192-202, 2016.
16. Lee S, Shin JH, Han BK and Ko EY: Medullary thyroid carcinoma: Comparison with papillary thyroid carcinoma and application of current sonographic criteria. AJR Am J Roentgenol 194: 1090-1094, 2010

17. Kim SH, Kim BS, Jung SL, Lee JW, Yang PS, Kang BJ, Lim HW, Kim JY, Whang IY, Kwon HS and Jung CK: Ultrasonographic findings of medullary thyroid carcinoma: A comparison with papillary thyroid carcinoma. Korean J Radiol 10: 101-105, 2009.

18. Trimboli P, Giovanella L, Valabrega S, Andrioli M, Baldelli R, Cremonini N, Rossi F, Guidobaldi L, Barnabei A, Rota F, et al: Ultrasound features of medullary thyroid carcinoma correlate with cancer aggressiveness: A retrospective multicenter study. J Exp Clin Cancer Res 33: 87, 2014.

19. Choi N, Moon WJ, Lee JH, Baek JH, Kim DW and Park SW: Ultrasonographic findings of medullary thyroid cancer: Differences according to tumor size andcorrelation with fine needle aspiration results. Acta Radiol 52: 312-316, 2011.

20. Ganeshan D, Paulson E, Duran C, Cabanillas ME, Busaidy NL and Charnsangavej $\mathrm{C}$ : Current update on medullary thyroid carcinoma. AJR Am J Roentgenol 201: W867-W876, 2013.

21. Cho KE, Gweon HM, Park AY, Yoo MR, Kim J, Youk JH, Park YM and Son EJ: Ultrasonographic features of medullary thyroid carcinoma: Do they correlate with Pre and PostOperative calcitonin levels? Asian Pac J Cancer Prev 17: 3357-3362, 2016

22. Woliński K, Rewaj-Łosyk M and Ruchała M: Sonographic features of medullary thyroid carcinomas-a systematic review and meta-analysis. Endokrynol Pol 65: 314-318, 2014.

23. Costante G, Meringolo D, Durante C, Bianchi D, Nocera M, Tumino S, Crocetti U, Attard M, Maranghi M, Torlontano M and Filetti S: Predictive value of serum calcitonin levels for preoperative diagnosis of medullary thyroid carcinoma in a cohort of 5817 consecutive patients with thyroid nodules. J Clin Endocrinol Metab 92: 450-455, 2007.

24. Chambon G, Alovisetti C, Idoux-Louche C, Reynaud C, Rodier M, Guedj AM, Chapuis H, Lallemant JG and Lallemant B: The use of preoperative routine measurement of basal serum thyrocalcitonin in candidates for thyroidectomy due to nodular thyroid disorders: Results from 2733 consecutive patients. J Clin Endocrinol Metab 96: 75-81, 2011.

25. Elisei R, Bottici V, Luchetti F, Di Coscio G, Romei C, Grasso L, Miccoli P, Iacconi P, Basolo F, Pinchera A and Pacini F: Impact of routine measurement of serum calcitonin on the diagnosis and outcome of medullary thyroid cancer: Experience in 10,864 patients with nodular thyroid disorders. J Clin Endocrinol Metab 89: 163-168, 2004.

26. Papapetrou PD and Polymeris A: Medullary thyroid carcinoma surgical cytoreduction induces an increase in serum calcitonin and carcinoembryonic antigen doubling times. Exp Clin Endocrinol Diabetes 120: 164-168, 2012.

27. Boschin IM, Torresan F, Toniato A, Zane M, Ide EC, Pennelli G, Rampin L, Colletti PM, Rubello D and Pelizzo MR: Incidental medullary thyroid microcarcinoma revealed by mild increase of preoperative serum calcitonin levels: Therapeutic implications. Endocrine 45: 448-453, 2014

28. Machens A, Hauptmann S and Dralle H: Prediction of lateral lymph node metastases in medullary thyroid cancer. Br J Surg 95: 586-591, 2008.

29. Rowland KJ, Jin LX and Moley JF: Biochemical cure after reoperations for medullary thyroid carcinoma: A meta-analysis. Ann Surg Oncol 22: 96-102,2015.

30. Rosario PW and Calsolari MR: Usefulness of serum calcitonin in patients without a suspicious history of medullary thyroid carcinoma and with thyroid nodules without an indication for fine-needle aspiration or with benign cytology. Horm Metab Res 48: 372-276, 2016.

This work is licensed under a Creative Commons Attribution-NonCommercial-NoDerivatives 4.0 International (CC BY-NC-ND 4.0) License. 